\title{
An Optimization Method for the Remanufacturing Dynamic Facility Layout Problem with Uncertainties
}

\author{
Lingling Li, ${ }^{1}$ Congbo Li, ${ }^{1}$ Huijie Ma, ${ }^{1}$ and Ying Tang ${ }^{2}$ \\ ${ }^{1}$ State Key Laboratory of Mechanical Transmission, Chongqing University, Chongqing 400030, China \\ ${ }^{2}$ Department of Electrical and Computer Engineering, Rowan University, Glassboro, NJ 08028, USA \\ Correspondence should be addressed to Congbo Li; cqulcb@163.com
}

Received 18 August 2014; Revised 15 October 2014; Accepted 11 November 2014

Academic Editor: Zhigang Jiang

Copyright (c) 2015 Lingling Li et al. This is an open access article distributed under the Creative Commons Attribution License, which permits unrestricted use, distribution, and reproduction in any medium, provided the original work is properly cited.

\begin{abstract}
Remanufacturing is a practice of growing importance due to increasing environmental awareness and regulations. Facility layout design, as the cornerstone of effective facility planning, is concerned about resource localization for a well-coordinated workflow that leads to lower material handling costs and reduced lead times. However, due to stochastic returns of used products/components and their uncontrollable quality conditions, the remanufacturing process exhibits a high level of uncertainty challenging the facility layout design for remanufacturing. This paper undertakes this problem and presents an optimization method for remanufacturing dynamic facility layout with variable process capacities, unequal processing cells, and intercell material handling. A dynamic multirow layout model is presented for layout optimization and a modified simulated annealing heuristic is proposed toward the determination of optimal layout schemes. The approach is demonstrated through a machine tool remanufacturing system.
\end{abstract}

\section{Introduction}

Remanufacturing, as an industrial process of restoring discarded products/components back to their useful lives, is of growing importance due to the emerging pressure of legislation and increasing awareness of environmental conservation. As the ultimate form of recycling, remanufacturing maintains much of the value added from original manufacturing to material and reprocessing conservation, leading to lower production costs and improved firm profits [1]. A recent Wall Street Journal revealed that large scale remanufacturing in the United States employs more than 500,000 people and contributes to approximately $\$ 100$ billion of goods sold each year [2]. More successful industry examples can be seen in Kodak, BMW, IBM, DEC, and Xerox [3]. However compared to manufacturing, remanufacturing is more complicated in the way that (1) the supply of returned products is unpredictable in timing and quantities; (2) the quality and composition of returned products vary; and (3) the process routings are not necessarily fixed but rather adapt to the actual conditions of products/components [4]. Such characteristics have led the facility layout problem (FLP) in remanufacturing to be much more complicated than that in traditional manufacturing.

Facility layout problem (FLP), as the cornerstone of effective facility planning, is concerned about resource localization for a well-coordinated workflow that directly or indirectly leads to lower material handling costs, less workin-process inventory, reduced lead times, and so forth [5]. Research in this area is commonly categorized as a static or dynamic FLP, depending on the nature of the input requirements and the time periods under consideration. Chaieb and Korbaa [6] dealt with the intracell machine layout problem in a circular bidirectional configuration to minimize both the movement distance and total transportation time. Chan et al. [7] incorporated the machine-part grouping decision into the static cellular layout problem to minimize the intercellular movement costs. Such efforts on static layout are usually performed for a single time period and assume flow between machines, product demand, and level of product mix constant.

Given the nature of manufacturing environments where uncertainty is inevitable, periodic layout reconfiguration with respect to changes is necessary as the best facility layout 
configuration for one time period may not be efficient for successive others. A relayout or dynamic facility layout problem (DFLP) is usually modeled by discretizing the time into several planning periods where the localization of an existing facility in each period is a decision variable. Dharmalingam et al. [8] proposed a cellular layout method for a manufacturing system with dynamic demands. With the assumptions of equal size cells and a fixed number of machines per cell, their objective is to minimize the number of intercell movements and to perform greatest proportion of part operations within a single cell. Sharing the same assumption that all departments have the same size, Baykasoglu et al. [9] developed a dynamic facility layout model to minimize both material handling and reconfiguration costs. In the authors' own claim, it was the first time that budget constraints were considered in DFLP. McKendall Jr. and Hakobyan [10] tackled DFLP with unequal-sized, free oriental departments. However, their design focused on open-field layout design with much loose constraints. By integrating the decisions of cell formation and group layout together, Kia et al. [11] developed a dynamic layout model that was incorporated with several design features, such as alternative process routings, operation sequences, machine capacity, lot splitting, multirow layout of equal area facilities, and flexible reconfigurations. The same group extended the work to propose a multiobjective model with more optimization constraints, including machine capability and capacity, part demand satisfaction, cell size, and location assignment [12].

Summarizing the findings of the above literatures, few efforts have been made towards the optimization of DFLP with consideration of remanufacturing natures. In fact, the high level of uncertainty in remanufacturing challenges its layout design to account for variations. For instance, the capacity of each remanufacturing process, the dimension of processing cells, and the scales of material handing between two cells are nonstationary but change with time. Thus, there is a strong case for focusing on DFLP in remanufacturing. In this paper, an attempt has been made to investigate such a problem. In particular, a dynamic multirow layout problem is studied with consideration of variable process capacities, unequal processing cells, and intercell material handling. The rest of the paper is organized as follows. Section 2 analyzes the uncertainties of DFLP in remanufacturing. Section 3 presents the dynamic facility layout optimization model. Section 4 presents a modified simulated annealing heuristic for the determination of optimal layout schemes. An example is provided in Section 5, followed by the conclusion in Section 6 .

\section{Uncertainties of FLP in Remanufacturing}

In a remanufacturing system, there are a number of uncertain characteristics that significantly complicate its remanufacturing activities $[4,13]$. Firstly, the product return process is highly uncertain with respect to timing, when the products are available for remanufacturing, and quantity, how many products are available. The uncertain characteristic in timing and quantity of returns is a reflection of the uncertain nature of the life of a product, which is commonly influenced by a number of factors including the life-cycle stage of a product and the rate of technological changes [14].

Product/component returned in remanufacturing exhibits highly uncontrolled variability with respect to product condition, ranging from slightly used with minor blemishes to significantly damaged and requiring extensive repair [14]. In a remanufacturing facility, used products are first manually disassembled into what is called "cores" that are disassembled parts or components. Then, inspection and sorting of returns are usually implemented in the system to identify core's quality and organize necessary activities for their recovery. According to inspection results, the cores are then classified into three groups (i.e., "reusable," "remanufacturable," and "material recoverable"). For the remanufacturable cores, their process routings are not fixed but rather adapted to their specific condition [15-17]. Figure 1 gives an example of the remanufacturing routings for cores (e.g., spindles, guide ways, worm gears, and lead screws) disassembled from a used machine tool. For instance, if a spindle is detected with severe abrasion, two typical process routings would be "grinding $\rightarrow$ chromium electroplating" or "grinding $\rightarrow$ cold welding $\rightarrow$ fine grind" to regain the surface accuracy. Among these routings, some remanufacturing operations may be probabilistic since the quality attributes of returned cores are characterized in the form of a set of classification probabilities [15].

Another uncertain characteristic associated with remanufacturing is the high degree of variability in remanufacturing processing times. For a failed core, the amount of time required to perform the necessary remanufacturing operation is strongly contingent upon the core's quality condition. In particular, the processing time is a function of the core, causing the core's failure, and the extent of the failure, which is unpredictable in prior.

From a facility layout perspective, the high level of uncertainty in remanufacturing challenges its layout design to account for variations. For instance, the remanufacturing processes are subject to extreme fluctuations in workloads because of uncertainty in stochastic returns, probabilistic process routings, and highly variable processing times. Accordingly, the required processing capacity of each remanufacturing process (i.e., the number of machines required to perform the specific remanufacturing operation) is nonstationary but changes with time. As a result, the dimensions of individual processing cells to accommodate a certain number of machines vary as well. Besides, due to the stochastic returns of used components and their stochastic process routings, the frequency of intercell material handling between two remanufacturing processing cells varies constantly over time. Given so much stochastic nature in remanufacturing, the best facility layout configuration obtained in a given time period may not be efficient for the successive others. Therefore, there is a strong case for focusing on DFLP in remanufacturing by discretizing the time into several planning periods. This paper undertakes this challenge and incorporates such variability in the optimization of a remanufacturing DFLP (R-DFLP) with the aim of achieving overall high efficiency and minimum cost. 


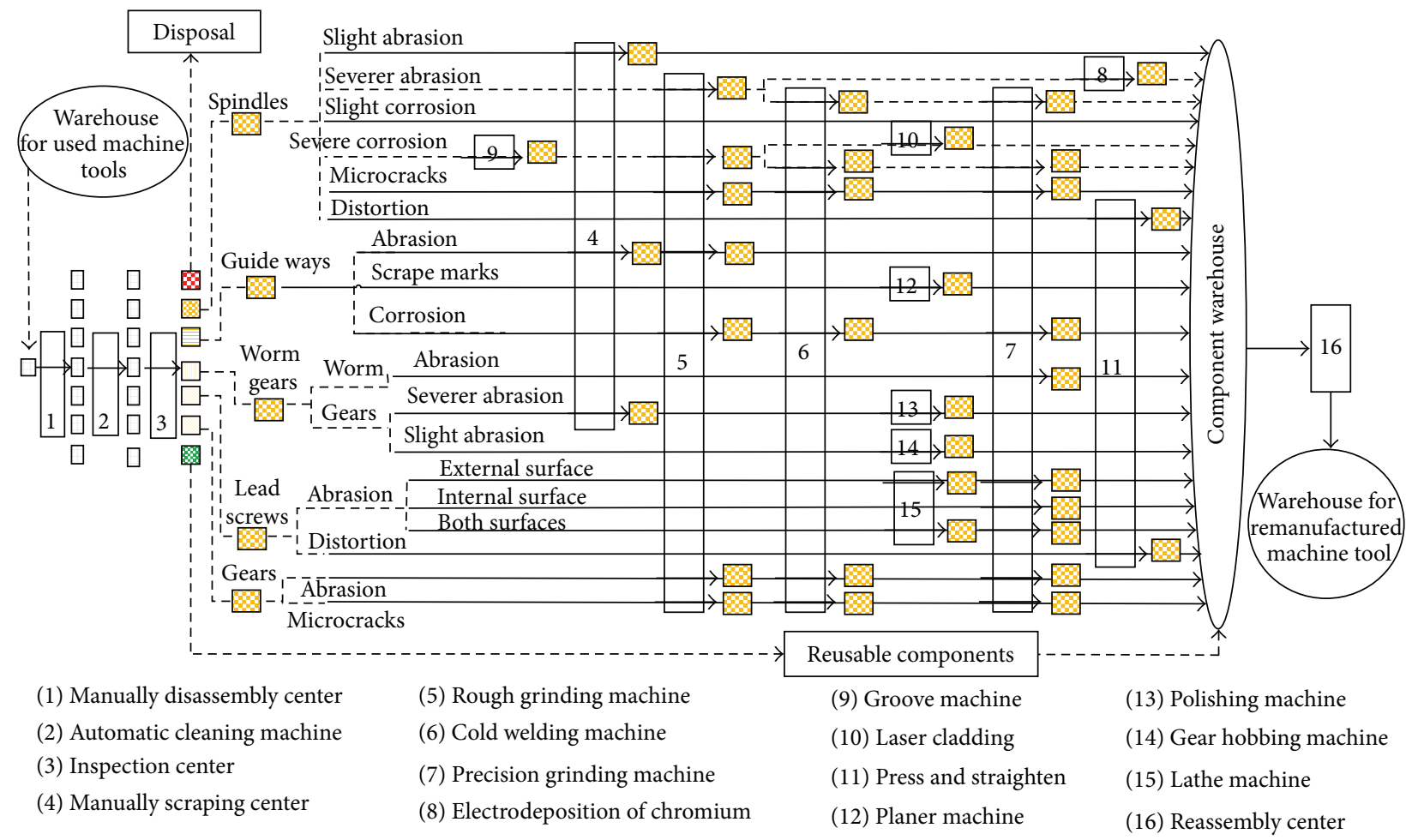

FIGURE 1: The remanufacturing process routings of machine tool cores.

\section{R-DFLP Optimization Model}

3.1. Problem Statement. A typical remanufacturing facility considered in the paper is a multirow shop floor, each row of which has a set of designated departments with an equal size. The facility accommodates a finite set of remanufacturing processes to restore components of a specific type of used products. A processing cell (i.e., grouping a number of machines to perform a specific remanufacturing operation) is dedicated to each remanufacturing process, which occupies one or more than one departments according to its dimension to be determined over time. Figure 2 gives an example of a facility layout configuration with 3 rows, 27 departments, and 6 processing cells. The problem is formulated under the following assumptions.

(a) A dynamic facility layout in remanufacturing is considered by discretizing the time into several planning periods $(t=1,2, \ldots, T)$. That is, the remanufacturing facility is allowed to be reconfigured at each given time period according to the dynamics of the system.

(b) The required capacity of a processing cell is not fixed but adapted to its workload in a given unit of time. Thus, the number of machines assigned to the cell might be different from one period to another. When $N_{i t}<N_{i(t-1)}$, the redundant machine would be removed from the cell. When $N_{i t}>N_{i(t-1)}$, additional machines will be added to the $i$ th processing cell. In either case, relocation cost would be charged for machine equipment handling regarding machine removal, machine transiting, and machine installation.

(c) There are an infinite number of machines in the facility. The purchase of new machines is not necessary and considered.

(d) The processing cells in the same row have the same $Y$-coordinate.

(e) Each processing cell only occupies departments in the same row.

(f) The dimension of the shop floor is given.

(g) The dimensions of machines are given but vary with respect to the processing cells they serve (i.e., the machines in a processing cell have the same dimensions).

(h) The intracell material handling cost is negligible.

(i) The width of a department is always larger than the length of machines in any processing cell.

(j) The orientations of machines in a processing cell are kept the same.

3.2. Notations and Variables Involved in R-DFLP. The notations and variables used in the model are given below.

\subsubsection{Index}

$t$ : time period index $(t=1,2, \ldots, T)$, where $T$ is the total number of periods; 


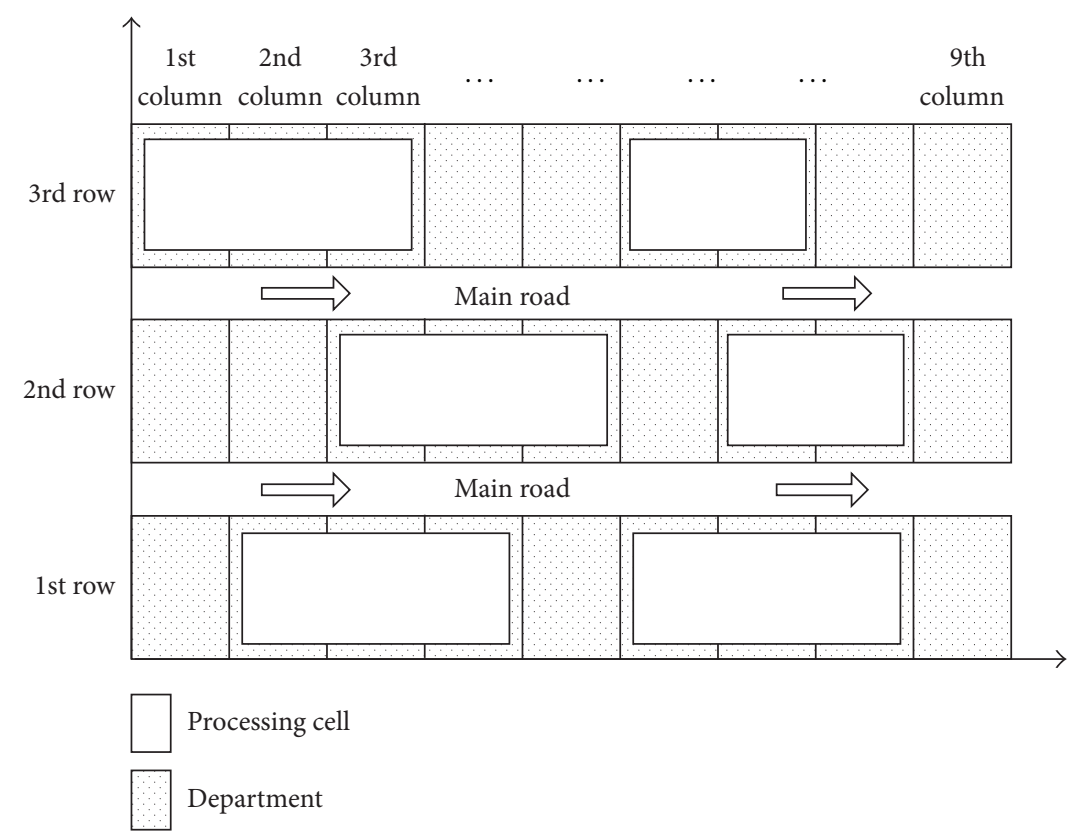

Figure 2: An example of a facility layout configuration with 3 rows, 27 departments, and 6 processing cells.

$i, j$ : processing cell index $(i, j=1,2, \ldots, Z)$, where $Z$ is the total number of processing cells in the shop floor; in particular, $i=1,2,3, Z$ denote disassembly, cleaning, inspection, and reassembly, respectively, while $i=4, \ldots, Z-1$ represent the remanufacturing operations;

$k$ : core type index;

$r$ : remanufacturing processing routing index;

$f$ : facility row index $(f=1,2, \ldots, F)$, where $F$ is the number of rows in the shop floor;

$h$ : department index in a given row $(h=1,2, \ldots, H)$, where $H$ is the number of departments per row.

\subsubsection{Model Parameters}

$D_{t}$ : time duration (in days) of the period $t$;

$Q_{k t}$ : number of the cores of type $k$ in period $t$;

$p_{k r t}$ : probability of the cores of type $k$ being assigned to the $r$ th processing routing in period $t$;

$c_{i}$ : machine availability (i.e., the available machine hours per workday of an individual machine) of the $i$ th processing cell (hours/day);

$\omega_{i}$ : machine efficiency (in percentage) of the $i$ th processing cell corresponding to machine failures;

$\psi_{i k}$ : average processing time (in minutes) for the cores of type $k$ in the $i$ th processing cell;

$M l_{i}$ : length of the machines in the $i$ th processing cell $\left(M l_{i} \geq M w_{i}\right)$

$M w_{i}$ : width of the machines in the $i$ th processing cell;
$S l$ : length of the remanufacturing facility $(S l \geq S w)$;

$S w$ : width of the remanufacturing facility;

$\varepsilon$ : the width of main road designed in the facility;

$\delta_{i}$ : relocation cost for the machines in the $i$ th processing cell;

$\theta_{k}$ : material handling cost of cores of type $k$ per unit of distances;

$\Delta_{t}$ : total material handling cost in period $t$;

$B_{t}$ : relocation budget in period $t$;

$\Gamma_{i j k r}$ : binary variable that has a value of 1 if the processing cell $i$ precedes the processing cell $j$ in the $r$ th processing routing for the cores of type $k$ and 0 otherwise;

$\alpha_{i k r}$ : binary variable that has a value of 1 if the $i$ th processing cell is in the $r$ th remanufacturing processing routing for the cores of type $k$ and 0 otherwise.

Among the above-mentioned parameters, there are three uncertain parameters that significantly complicate the RDFLP and need to be elaborated as follows.

(1) The Arrivals of Used Products. In a remanufacturing system, arrivals $\left(Q_{k t}\right)$ related to the failures of products are uncertain in timing and quantities and are commonly modelled as a Poison process [9].

(2) The Process Routings. The process routings are not necessarily fixed but rather adapt to the actual conditions of cores. The likelihood $\left(P_{k r t}\right)$ of a remanufacturing process routing being assigned to a certain type of cores is randomly generated to represent the stochastic features [4]. 
(3) The Processing Times. The processing times $\left(\psi_{i k}\right)$ required to perform the necessary remanufacturing operations for a certain part are highly variable. An exponential distribution is used for the processing times in order to simulate the large range of possible values [9].

\subsubsection{Decision Variables}

$N_{i t}$ : number of machines in the $i$ th processing cell in period $t$;

$\beta_{i t}$ : binary variable that has a value of 1 if the machines in the $i$ th processing cell are oriented vertically and 0 otherwise;

$L_{i t}$ : length of the $i$ th processing cell in period $t$;

$\eta_{i t}$ : number of departments occupied by the $i$ th processing cell in period $t$;

$\pi_{\text {ihft }}$ : binary variable that has a value of 1 if the $i$ th processing cell occupies the department in the $h$ th row and $i$ th column as its first location in period $t$;

$\left(x_{i t}, y_{i t}\right)$ : the centroid of the $i$ th processing cell in period $t$;

$d_{i j t}$ : distance (in meters) between the two processing cells $i$ and $j$ in period $t$;

$\tau_{i t}$ : binary variable that has a value of 1 if the centroid of the processing cell $i$ in period $t$ is different from that in period $t-1$ and 0 otherwise.

As stated earlier, the variability in remanufacturing regarding stochastic returns, probabilistic process routings, and highly variable processing times complicates its facility layout design. Thus the decision variables in the R-DFLP, including the number of machines, the dimensions of individual processing cells, and the detailed location (i.e., the centroid) of each processing cell in a given time period, will be mathematically elaborated in the following.

(1) Number of Machines in Processing Cells. The number of machines dedicated to the $i$ th processing cell in period $t, N_{i t}$, is a function of the number of cores needed to be processed at the time, the average processing times, and machine availability and efficiency:

$$
N_{i t}=\frac{\sum_{k} \sum_{r} Q_{k t} \cdot p_{k r t} \cdot \psi_{i k} \cdot \alpha_{i k r}}{\omega_{i} \cdot c_{i} \cdot D_{t}},
$$

where $c_{i}$ denotes the machine availability (i.e., the available machine hours per workday of a certain machine type) of the $i$ th processing cell (hours/day) and $\omega_{i}$ is the machine efficiency (in percentage) of the $i$ th processing cell corresponding to machine failures.

(2) Dimensions of Processing Cells. It is assumed that an individual processing cell should be equipped with a series of machines of the same type to perform a specific remanufacturing process. The dimension of a processing cell as formulated in (2) is pertinent to the numbers of machines dwelt in and their orientations. The number of departments occupied by the $i$ th processing cell is given in (3):

$$
\begin{gathered}
L_{i t}=\left[M l_{i} \cdot\left(1-\beta_{i t}\right)+M w_{i} \cdot \beta_{i t}\right] \cdot N_{i t}, \\
\eta_{i t}=\text { ceil }\left[\frac{L_{i t}}{S l / H}\right],
\end{gathered}
$$

where $S l / H$ (i.e., dividing $S l$ by $H$ ) gets the length of each department designed in each row of the floor shop and ceiling $(x)$ returns a value that is the smallest integer not less than $x$.

(3) The Centroids of Processing Cells. For each processing cell, with the required number of departments $\left(\eta_{i t}\right)$ and the first department it occupies $\left(\pi_{\text {ihft }}\right)$ being determined, its centroids in period $t$ then can be determined by (4). The distance between the centroids of the processing cell $i$ and $j$ in period $t$ is given in (5):

$$
\begin{gathered}
x_{i t}=(h-0.5) \cdot \frac{S l}{2 H}+\left(\eta_{i t}-1\right) \cdot \frac{S l}{2 H}, \\
y_{i t}=\frac{S w}{2 F}+(f-1) \cdot \frac{S w}{F}, \\
\forall \pi_{i h f t}=1, \\
d_{i j t}=\left|x_{i t}-x_{j t}\right|+\left|y_{i t}-y_{j t}\right| .
\end{gathered}
$$

3.3. Optimization Model. The R-DFLP considered in this paper aims to balance the trade-off between the intercell material handling cost and the increased relocation cost of inefficient layouts.

(1) Cost of Intercell Material Handing. While cores go through various processes to be restored back to their useful lives, material handling cost is associated with their logistic activities (i.e., moving from one processing cell to another). The total amount of material handling cost in a given unit of time is an incremental linear function of the total distances of cores moving in the facility, as defined below:

$$
\Delta_{t}=\sum_{k} \sum_{r} \sum_{i} \sum_{j} Q_{k t} \cdot p_{k r t} \cdot \Gamma_{i j k r} \cdot d_{i j t} \cdot \theta_{k},
$$

where $\theta_{k}$ denotes the material handling cost of cores of type $k$ per unit distance.

(2) Relocation Cost. Since the best facility layout scheme for one time period may not be efficient for the successive others, the floor shop needs to be reconfigured accordingly by changing the locations of a series of processing cells in a given time period. In this case, machine equipment in these processing cells needs to be removed, transited, 
and reinstalled which necessarily incur a certain amount of relocation costs, as formulated in the following:

$$
\begin{gathered}
\Omega_{t}=\sum_{i} \tau_{i t} \cdot N_{i t} \cdot \delta_{i}, \quad \forall t>1, \\
\tau_{i t}= \begin{cases}0, & \text { if } x_{i t}=x_{i(t-1)}, y_{i t}=y_{i(t-1)}, \quad \forall t>1, \\
1, & \text { otherwise, }\end{cases}
\end{gathered}
$$

where $\tau_{i t}$ is a binary variable that has a value of 1 if the centroid of the processing cell $i$ in period $t$ is different from that in period $t-1$ and 0 otherwise; $\delta_{i}$ is the relocation cost per machine of the $i$ th processing cell.

The R-DFLP problem is then formulated as a nonlinear mixed-integer programming model to minimize the total cost of intercell material handling and machine relocation:

$$
\begin{gathered}
\text { Minimize total cost }=\sum_{t=1}^{T} \Delta_{t}+\sum_{t=2}^{T} \Omega_{t} \\
\text { Subject to } \quad \sum_{t=2}^{T} \sum_{i} \tau_{i t} \cdot \delta_{i} \leq B_{t} \\
\sum_{i} \eta_{i t} \leq F \cdot H, \quad \forall t, \\
\sum_{i=3} \sum_{j=4}^{Z-1} \sum_{k} \sum_{r} Q_{k t} \cdot p_{k r t} \cdot \Gamma_{i j k r} \\
=\sum_{i=4}^{Z-1} \sum_{j=Z} \sum_{k} \sum_{r} Q_{k t} \cdot p_{k r t} \cdot \Gamma_{i j k r}, \quad \forall t .
\end{gathered}
$$

Constraint (9) assures that the total relocation cost in any period does not exceed the given budget. Constraint (10) guarantees that the total number of departments occupied by processing cells is not larger than the total available in the shop floor. Constraint (11) represents material flow conservation between disassembly and reassembly.

\section{The Simulated Annealing Algorithm for R-DFLP}

Simulated annealing (SA) is a stochastic gradient method for global optimization that has been successfully applied to a wide variety of sophisticated combinatorial problems [18-20]. While it conducts a local search, it is capable of exploring the solution space stochastically and effectively to prevent being trapped in a local optimum. The SA procedure starts from an initial basic solution $s_{0}$ and generates a neighbourhood solution $s^{\prime}$ by a suitable mechanism. To accept $s^{\prime}$ over $s_{0}, s^{\prime}$ either is a better neighbourhood solution or has a certain probability to escape from a local minimum though $s^{\prime}$ is worse than $s_{0}$ [21]. The acceptance probability AP is often defined as follows:

$$
\mathrm{AP}= \begin{cases}1, & \text { if } \Lambda<0, \\ \exp \left\{\frac{-\Lambda}{\Omega}\right\}, & \text { otherwise }\end{cases}
$$

where $\Lambda=f\left(s^{\prime}\right)-f\left(s_{0}\right), \Omega$ is called temperature, a global time-varying parameter, and $f()$ is the optimization objective function. At each iteration, the parameter $\Omega$ is reduced by a factor $\vartheta$ and the chance of choosing an inferior solution decreases as well. The search process, which is generating a neighbour solution, rejecting or accepting it with the above probability, continues until the stopping criterion is met. Given SA is a well-established metaheuristic, the major steps are modified to suit our specific application, followed by the proposed algorithms.

4.1. Solution Representation. To address the challenge of RDFLP with unequal processing cells, this paper proposes a special solution representation that uses two-stage matrices for remanufacturing layout schemes.

The first matrix $A=\left[a_{t w}\right]_{T \times W}$ is called sequence matrix that defines the sequence of processing cells to be allocated departments in any given period, where $W=Z+F \cdot H-$ $\sum \eta_{i t} . a_{t w}$ is a nonnegative integer, indicating the index of a processing cell that is the $w$ th in sequence to be assigned departments in period $t$. If $a_{t w}=0$, a department is left unoccupied in the $w$ th action. For each unoccupied department, an intangible machine is assumed to be allocated in it. That is, $\forall a_{t w}=0$, and there exists $N_{\left[a_{t w}\right] t}=1$, $M l_{\left[a_{t w}\right]}=S L / H$, and $M w_{\left[a_{t w}\right]}=[S W-(F-1) \varepsilon] / F$. Figure 3(a) gives an example of $A$ that represents allocation sequences in four time periods. For instance, in the first time period, the allocation order is the processing cells $1,3,2$, and 4 , followed by an unoccupied department.

The second matrix $E=\left[e_{t w}\right]_{T \times W}$ has the same dimensions as matrix $A$ that defines the orientation of processing cells in period $t . e_{t w}$ takes a binary value of zero or one, indicating the processing cell in the $w$ th allocation sequence is oriented vertically (1) or horizontally (0). In this case, matrix $E$ is determined according to matrix $A$ as defined in (13). It should be noted that if $a_{t w}>0$, then the machine can be located either vertically or horizontally (i.e., $e_{t w} \in\{0,1\}$ ). If $a_{t w}=0$, then $e_{t w}=1$ which denotes that the intangible machine can only be oriented vertically in the unoccupied department. Figure 3(b) gives an example of matrix $E$ that aligns to matrix $A$ in Figure 3(b):

$$
e_{t w}= \begin{cases}\beta_{\left[a_{t w}\right] t} \in\{0,1\}, & a_{t w}>0 \\ 1, & a_{t w}=0\end{cases}
$$

To validate the generated solutions, another matrix, $G=$ $\left[g_{f h t}\right]_{F \times H \times T}$, is defined to provide the detailed layout scheme for all processing cells in remanufacturing facility. $g_{f h t}$ is the index of the processing cell that occupies the department in the $f$ th row and $h$ th column in period $t$. If $g_{f h t}=0$, the department in the $f$ th row and the $h$ th column is unoccupied. Matrix $G$ is not part of the solution representation but can be directly derived from matrices $A$ and $E$. Given the matrices $A$ and $E, L_{i t}$ and $\eta_{i t}$ can be first calculated, and then matrix $G$ can be determined according to Procedure 1. A sample layout matrix is provided in Figure 3(b) that is directly derived from the sample matrix $A$ in Figure 3(a). 


$$
A=\left[\begin{array}{lllll}
1 & 3 & 2 & 4 & 0 \\
2 & 1 & 3 & 0 & 4 \\
1 & 0 & 2 & 4 & 3 \\
1 & 2 & 0 & 3 & 4
\end{array}\right] \quad E=\left[\begin{array}{lllll}
1 & 0 & 0 & 0 & 1 \\
1 & 0 & 1 & 1 & 0 \\
1 & 1 & 0 & 1 & 0 \\
1 & 0 & 1 & 0 & 1
\end{array}\right]
$$

(a) (b)

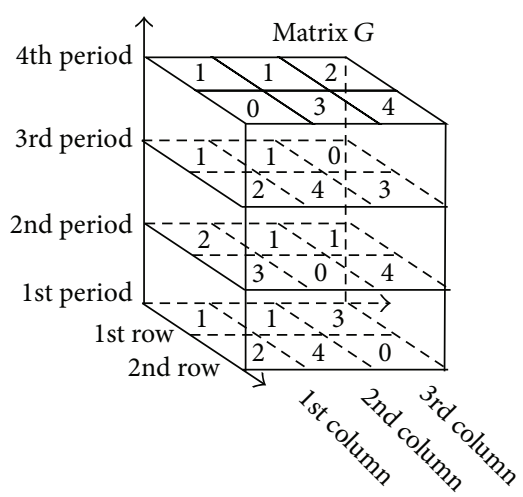

(c)

Figure 3: (a) Sample matrix $A$; (b) sample matrix $E$; (c) sample matrix $G$ derived from $A$ and $E$.

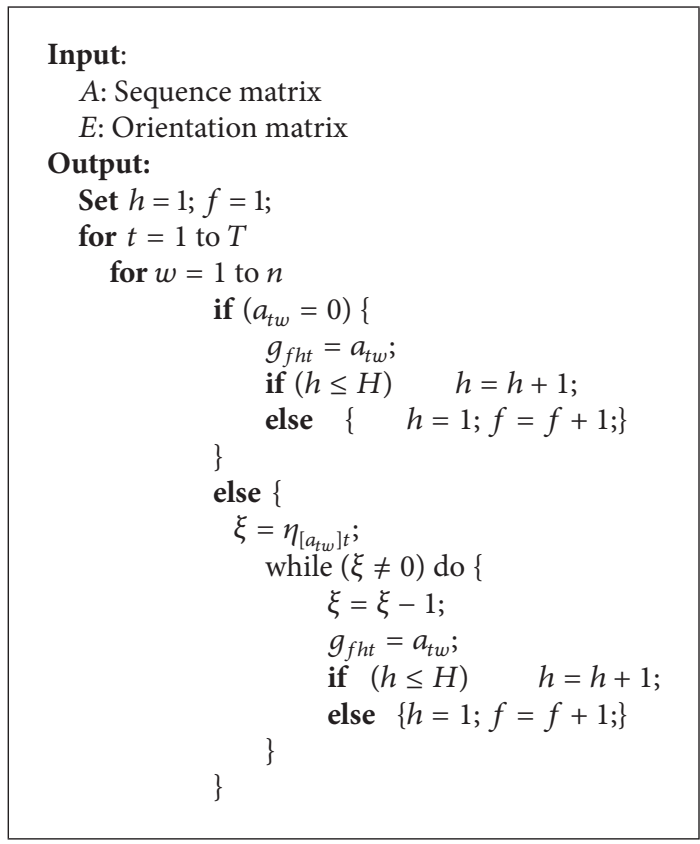

Procedure 1: Determination of the layout matrix $G$.

For each processing cell, with its dimension (i.e., $L_{i t}$, $\left.\eta_{i t}\right)$ and allocation sequence (i.e., in matrix $A$ ) determined, Procedure 1 first selects a corresponding department as its starting location. The selection continues with the right neighbours of the departments from the left to the right until the dimension of the processing cell is met. With the way of allocating departments to processing cells and the assumption that each processing cell only occupies departments in the same row, the layout matrix should meet the following condition:

$$
g_{f H t} \neq g_{(f+1) 1 t}, \quad \forall f=\{1,2, \ldots, F-1\} .
$$

With a feasible matric $G$ obtained, the first department that the $i$ th processing cell occupies can be determined by (15). Then using (4)-(8) the total cost can be calculated accordingly:

$$
\pi_{\text {ihft }}=\left\{\begin{array}{ll}
1, & \forall f=\min _{\forall g_{\text {fht }}=i}\{f\}, \\
0, & \text { otherwise, }
\end{array} \quad \forall i, t .\right.
$$

4.2. Initial Solution Generation. The initial solution is important to the performance of SA as the quality of the final solution relies heavily on that of the initial solution [22, 23]. The procedure of generating an initial solution is given below.

\subsection{Procedure: SA Initialization}

(1) Generate two sets, the acceptable solution set AS = $\left\{\left(A_{v}, E_{v}\right), v=1,2, \ldots, O\right\}$ and the invalid solution set IS $=\left\{\left(A_{u}, E_{u}\right)\right\}$. Initialize AS and IS to be empty.

(2) Randomly generate a solution, 2-tuple $\left\{\left(A^{*}, E^{*}\right)\right\}$. If $\left(A^{*}, E^{*}\right) \notin$ IS, go to (3); otherwise, restart (2).

(3) Derive the layout matrix $G^{*}$ based on $\left(A^{*}, E^{*}\right)$. If $G$ satisfies constraints (6) and (10), AS $=\operatorname{AS} \cup\left(A^{*}, E^{*}\right)$. If $|\mathrm{AS}|=O$, go to (4); otherwise, IS = IS $\cup\left(A^{*}, E^{*}\right)$, go back to (2).

(4) Calculate the total cost of each feasible solution in AS, and choose the one with the minimal total cost as the initial solution $s_{0}$ for the SA algorithm (Algorithm 1).

4.4. Neighbourhood Solution Generation. In order to obtain the neighbourhood solution from the current solution, the standard SA neighbourhood structure that features various types of move in work [23] is adopted. The detailed procedure is given below.

\subsection{Procedure: Neighbour_S(s)}

(1) Randomly select a row $t$ and a column $w$ in matrix $A$, and use one type of moves (i.e., $m$-opt, $m \in$ $\{1,2, \ldots, n-w\})$ to exchange $a_{t w}$ with $a_{t(w+m)}$ and formulate a new matrix $A^{\prime}$. Then randomly select a $e_{t w}>0$ in matrix $E$, and update $e_{t w}=1-e_{t w}$ 
Step 1. Set the annealing parameters: $\Omega$ and $\vartheta$ and initialize the outer loop counter (ol) and the inner loop counter (il) zero. $\mathrm{ol}_{\max }, \mathrm{il}_{\max }$, and $\sigma_{\max }$ are maximum outer loop, inner loop, and identical solution, respectively.

Step 2. Apply Procedure SA initialization to generate an initial solution $s_{0}$. Assign $s_{\mathrm{ol}}=s=s_{0}$.

Step 3. While (ol $\leq \mathrm{ol}_{\max }$ and $\sigma \leq \sigma_{\max }$ ) do:

for $\left(\mathrm{il}=0\right.$; $\left.\mathrm{il} \leq \mathrm{il}_{\max } ; \mathrm{il}=\mathrm{il}+1\right)$

Apply Procedure Neighbour_S $(s)$

Calculate $\Lambda=f\left(s^{\prime}\right)-f(s)$

If $(\Lambda<0)$ or $(\exp \{-\Lambda / \Omega\} \geq \operatorname{Random}(0,1))$

$s=s^{\prime}$

else reject $s^{\prime}$

$\mathrm{ol}=\mathrm{ol}+1$

$s_{\mathrm{ol}}=s$;

if $\left(s_{\mathrm{ol}}=s_{(\mathrm{ol}-1)}\right) \quad \sigma=\sigma+1$;

else $\quad \sigma=0$;

Step 4. Output the matrix $G$ based on $s_{\mathrm{ol}}$

Algorithm 1: Proposed SA.

to formulate a new matrix $E^{\prime}$. A potential neighbour solution $s^{\prime}=\left(A^{\prime}, E^{\prime}\right)$ is then formulated.

(2) Output $s^{\prime}$ as the neighbourhood solution if it satisfies the constraints (6) and (10); otherwise, go back to (1).

\section{An Example}

In this section, a sample machine tool remanufacturing facility with 2 rows and 16 processing cells is used to demonstrate the application of the proposed model and algorithm. The workflows of cores in this remanufacturing shop are shown in Figure 2. In order to verify the efficiency of the proposed method, two cases are considered in the simulation for a 12 -month period. In the static case, the system treats the 12 months as a single period and no reconfiguration is allowed. In the dynamic case, the facility layout is reconfigured at every 4 months based on the changes in the supplier. The proposed SA algorithm is applied to both cases using Matlab 2009. During the implementation of the simulation, the input data is populated as follows. The facility data is shown in Table 1.

(1) The arrival of used machine tools satisfies a Poisson distribution. Thus the arrival of each type of remanufacturable cores in period $t$ follows a Poisson distribution with the arrival rate, $\lambda \in U[10,15] * \pi$, where $\pi \in\{1.0,1.1,1.2,1.3,1.4,1.5\}$ is the arrival rate expansion factor.

(2) The probabilities of each type of cores going through various process routings in a certain period are randomly generated $p_{k r t} \in[0,1]$ and $\sum_{r} p_{k r t}=1$.

(3) The average processing time for the cores of type $k$ in the $i$ th processing cell follows an exponential distribution, $\psi_{i k} \sim \Gamma(1, \lambda)$, where $\lambda \in[0.08,0.10]$.

Figure 4 compares the total cost of the dynamic layout scheme to that of the static one via twenty-four runs of the SA where the relocation cost $\delta=100$ and $\pi=1.0$. It is clear that the dynamic layout scheme outperforms the static one with about $20 \%$ cost reduction. Figure 5 shows the optimal layout
TABLE 1: Other data involved in the dynamic facility layout problem.

\begin{tabular}{ll}
\hline$S l=85 \mathrm{~m}$ & $S w=20 \mathrm{~m}$ \\
$F=2$ & $H=16$ \\
$c_{i}=8$ hours/day & $\omega_{I}=95 \%$ \\
$B_{1}=B_{2}=B_{3}=B_{4}=1000$ & $\theta_{1}=\theta_{3}=\theta_{4}=\theta_{5}=1, \theta_{2}=5$ \\
$\delta_{i}=100, \forall i \in\{1,2, \ldots, 16\}$ & $\oplus=80 \%$ \\
$\psi_{1 k}=\psi_{2 k}=15 \mathrm{~min}$ & $\psi_{3 k}=5 \mathrm{~min} ; \psi_{16, k}=20 \mathrm{~min}$ \\
$M l_{1}=M l_{2}=M l_{16}=$ & $M w_{1}=M w_{2}=M w_{16}=$ \\
$M l_{14}=M l_{12}=M l_{6}=$ & $M w_{14}=M w_{12}=3 \mathrm{~m}$ \\
$M l_{8}=M l_{10}=6 \mathrm{~m}$ & $M w_{6}=M w_{8}=M w_{10}=5 \mathrm{~m}$ \\
$M l_{i}=3 \mathrm{~m}, \forall i \in\{4,5,6, \ldots\}$ & $M w_{i}=2 \mathrm{~m}, \forall i \in\{4,5,6, \ldots\}$ \\
\hline
\end{tabular}

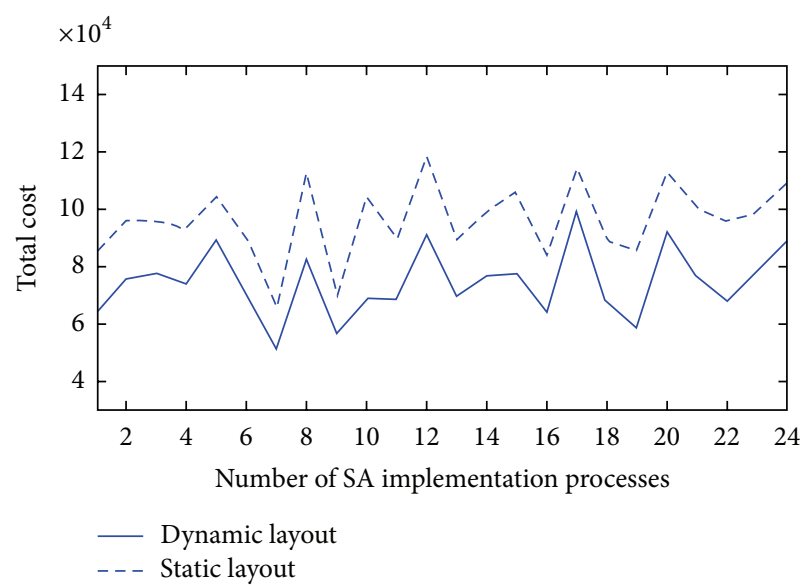

Figure 4: Performance comparison of the dynamic layout versus the static one $(\delta=100, \pi=1.0)$.

schemes for both dynamic layout and static one derived from the 8 th simulation. Table 2 gives the detailed indication of the letters A-T in Figures 5 and 6.

In order to determine any significance difference between performances of the dynamic layout and the static one, a set of simulations are implemented for different arrival rate level. 


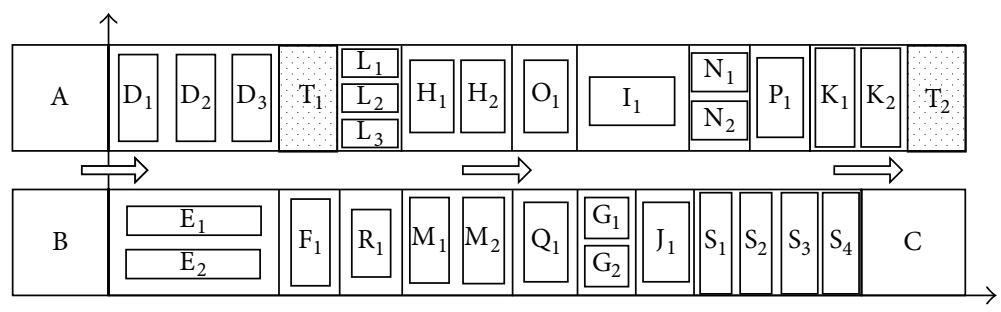

(a) The dynamic layout scheme for 1-4 months (material handling cost $=23224$ )

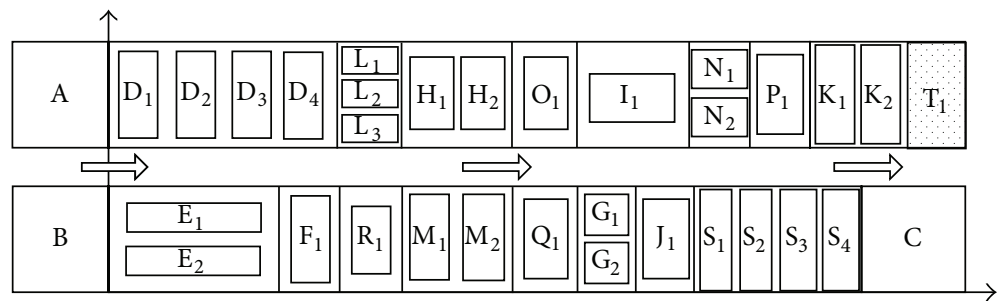

(b) The dynamic layout scheme for 5-8 months (total cost $=32148$ )

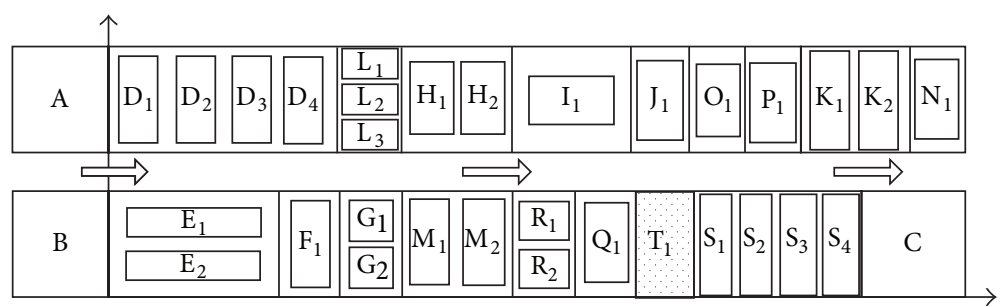

(c) The dynamic layout scheme for 9-12 months (total cost $=27663$ )

FIGURE 5: The dynamic layout scheme for a whole year (total cost $=83035)$.

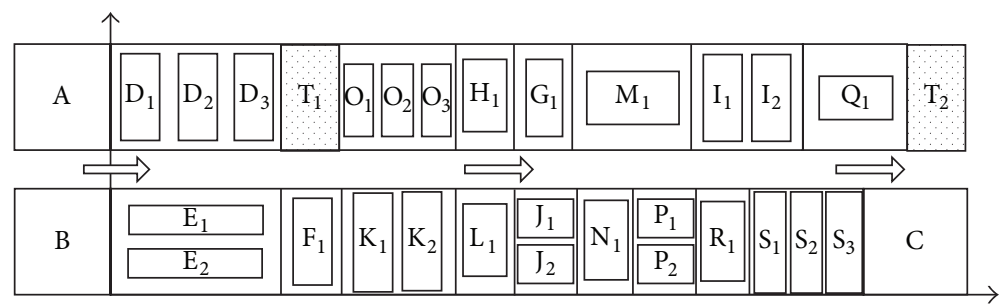

FIgURE 6: The static layout scheme for a whole year (total cost $=113250)$.

TABLE 2: The detailed indication of the letters A-T in Figures 5 and 6.

\begin{tabular}{|c|c|c|c|c|c|c|c|}
\hline $\mathrm{A}-\mathrm{E}$ & Indication & F-J & Indication & $\mathrm{K}-\mathrm{O}$ & Indication & $\mathrm{P}-\mathrm{T}$ & Indication \\
\hline A & $\begin{array}{l}\text { Warehouse for used } \\
\text { machine tool }\end{array}$ & $\mathrm{F}$ & Inspection center & $\mathrm{K}$ & Chromium electroplating & $\mathrm{P}$ & Polishing machine \\
\hline B & Workshop office & G & Manually scraping center & $\mathrm{L}$ & Groove machine & Q & Gear hobbing machine \\
\hline $\mathrm{C}$ & $\begin{array}{l}\text { Warehouse for } \\
\text { remanufactured } \\
\text { machine tool }\end{array}$ & $\mathrm{H}$ & Rough grinding machine & M & Laser cladding & $\mathrm{R}$ & Lathe machine \\
\hline $\mathrm{D}$ & $\begin{array}{l}\text { Manually disassembly } \\
\text { center }\end{array}$ & I & Cold welding machine & $\mathrm{N}$ & Press and straighten & S & Reassembly center \\
\hline $\mathrm{E}$ & $\begin{array}{c}\text { Automatic cleaning } \\
\text { machine }\end{array}$ & $\mathrm{J}$ & Precision grinding machine & $\mathrm{O}$ & Planer machine & $\mathrm{T}$ & Unoccupied department \\
\hline
\end{tabular}


TABlE 3: Comparison of the average total cost of two cases.

\begin{tabular}{|c|c|c|c|c|}
\hline \multirow[b]{2}{*}{ Arrival rate level } & \multicolumn{2}{|c|}{ The average total cost } & \multirow[b]{2}{*}{$\begin{array}{c}\text { Cost difference } \\
\phi_{2}\end{array}$} & \multirow[b]{2}{*}{$\begin{array}{l}\text { Cost reduction ratio } \\
\qquad\left(\phi_{1}-\phi_{2}\right) / \phi_{1}\end{array}$} \\
\hline & $\begin{array}{c}\text { The static layout } \\
\phi_{1}\end{array}$ & $\begin{array}{l}\text { The dynamic layout } \\
\phi_{2}\end{array}$ & & \\
\hline$v=1.0$ & 100915 & 78084 & 28831 & 0.226239 \\
\hline$v=1.1$ & 124947 & 90250 & 34697 & 0.277694 \\
\hline$v=1.2$ & 148068 & 109396 & 38672 & 0.261177 \\
\hline$v=1.3$ & 178295 & 135050 & 43245 & 0.242547 \\
\hline$v=1.4$ & 196572 & 147063 & 49509 & 0.251862 \\
\hline$v=1.5$ & 233200 & 178090 & 55110 & 0.236321 \\
\hline Sample mean & 163666.2 & 122988.8 & 41677.33 & 0.249307 \\
\hline Sample standard deviation & 48604.4 & 37507.91 & 9664.517 & 0.018437 \\
\hline
\end{tabular}

The average total cost is obtained and compared among the dynamic layout and the static one, as shown in Table 3. It is clear to see that the dynamic layout scheme outperforms the static one in terms of generating less cost. A 95\% confidence interval for the cost reduction ratio of the dynamic layout is also conducted using the data in Table 3. The approximate $100(1-\alpha) \%$ confidence interval for $\phi$ is defined as

$$
\begin{aligned}
& \widehat{\phi} \pm t_{1-\alpha / 2}(n-1) \frac{S}{\sqrt{n}}, \\
& \text { or } \widehat{\phi}-t_{1-\alpha / 2}(n-1) \frac{S}{\sqrt{n}} \leq \phi \leq \widehat{\phi}-t_{1-\alpha / 2}(n-1) \frac{S}{\sqrt{n}},
\end{aligned}
$$

where $\widehat{\phi}$ is the sample mean of $\phi$ based on a sample of size $n$; $S$ is the sample standard deviation; $t_{1-\alpha / 2}(n-1)$ is the $100(1-$ $\alpha) \%$ percentage point of a $t$-distributed with $n-1$ degree of freedom.

A 95\% confidence interval for the cost reduction ratio of the dynamic layout is given by

$$
\begin{aligned}
& 0.249307 \pm\left(2.571 \times \frac{0.018437}{\sqrt{6}}\right) \\
& 0.229955 \leq \frac{\phi_{1}-\phi_{2}}{\phi_{1}} \leq 0.268659 .
\end{aligned}
$$

The $95 \%$ confidence interval for the cost reduction ratio lies completely above zero, that is, $\left(\phi_{1}-\phi_{2}\right) / \phi_{1}>0$, which provides strong evidence that at $95 \%$ confidence there is significant difference between performances of the dynamic layout and the static one.

The similar comparison is conducted with the relocation cost changing from 100 to 1900 . Figure 7 shows that when relocation cost is less than 1200 , relocation of machines in dynamic layout would lead to much reduction in material handling and is significant to lower the overall cost. However, when relocation becomes extremely expensive (i.e., higher than 1200), the dynamic layout starts to lose its advantage. The trends in Figure 7 indicate that the dynamic layout is much superior to the static one especially for the facilities with higher flexibility in machine equipment handling. However if the facility has gained much resistance to machine equipment handling, the static layout would outperform the dynamic one with less cost.

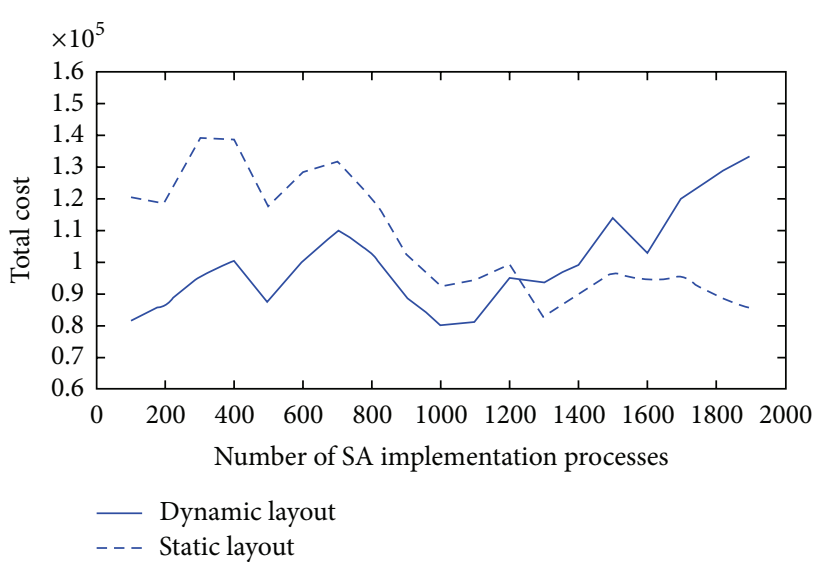

Figure 7: Performance of the dynamic layout schemes and the static ones with relocation cost ranging from 100 to 1900.

\section{Conclusion}

The remanufacturing process exhibits a high level of uncertainty due to stochastic returns of used products/components and their uncontrollable quality. Such uncertainty challenges the facility layout design for remanufacturing. This paper undertakes this problem and presents an optimization method for remanufacturing dynamic layout design with variable process capacities, unequal processing cells, and intercell material handling. The uncertainties inherent in the remanufacturing facility layout are explicitly analyzed. A dynamic multirow layout model is present for layout optimization with minimization of both material handling cost and machine relocation cost. A modified simulated annealing heuristic is proposed towards the determination of optimal layout schemes with unequal processing cells. The approach is validated through a layout design for a machine tool remanufacturing system and its effectiveness is demonstrated through simulations. Through simulations it is shown that while relocation cost incurs in dynamic layout reconfiguration, the reduction in material handling is significant to lower the overall cost. However, when relocation becomes extremely expensive, the dynamic layout starts to lose its advantage. In the future, factory date will 
be used to test our methodology through more complicated industrial cases.

\section{Conflict of Interests}

The authors declare that there is no conflict of interests regarding the publication of this paper.

\section{Acknowledgments}

This work was supported in part by the National Key Technology R\&D Program of China (2012BAF02B03) and the National Natural Science Foundation of China (51105395).

\section{References}

[1] T. Guidat, A. P. Barquet, H. Widera, H. Rozenfeld, and G. Seliger, "Guidelines for the definition of innovative industrial product-service systems (PSS) business models for remanufacturing," in Proceedings of the 6th CIRP Conference on Industrial Product Service Systems, pp. 193-198, Ontario, Canada, May 2014.

[2] A. Atasu, M. Sarvary, and L. N. V. Wassenhove, "Remanufacturing as a marketing strategy," Management Science, vol. 54, no. 10, pp. 1731-1746, 2008.

[3] J. Östlin, E. Sundin, and M. Björkman, "Product life-cycle implications for remanufacturing strategies," Journal of Cleaner Production, vol. 17, no. 11, pp. 999-1009, 2009.

[4] Y. Tang and C. B. Li, "Uncertainty management in remanufacturing: a review," in Proceedings of the 8th IEEE International Conference on Automation Science and Engineering, pp. 52-57, Seoul, Republic of Korea, August 2012.

[5] R. Sahin, K. Ertogral, and O. Turkbey, "A simulated annealing heuristic for the dynamic layout problem with budget control," Computers and Operations Research, vol. 59, no. 2, pp. 308-313, 2010.

[6] I. Chaieb and O. Korbaa, "Intra-cell machine layout associated with flexible production and transport systems," Proceedings of the Institution of Mechanical Engineers, Part B: Journal of Engineering Manufacture, vol. 217, no. 7, pp. 883-892, 2003.

[7] F. T. S. Chan, K. W. Lau, P. L. Y. Chan, and K. L. Choy, "Two-stage approach for machine-part grouping and cell layout problems," Robotics and Computer-Integrated Manufacturing, vol. 22, no. 3, pp. 217-238, 2006.

[8] P. Dharmalingam, K. Kanthavel, R. Sathiyamoorthy, M. Sakthivel, R. Krishnaraj, and C. Elango, "Optimization of cellular layout under dynamic demand environment by simulated annealing," International Journal of Scientific and Engineering Research, vol. 3, no. 10, pp. 91-97, 2012.

[9] A. Baykasoglu, T. Dereli, and I. Sabuncu, "An ant colony algorithm for solving budget constrained and unconstrained dynamic facility layout problems," Omega, vol. 34, no. 4, pp. 385-396, 2006.

[10] A. R. McKendall Jr. and A. Hakobyan, "Heuristics for the dynamic facility layout problem with unequal-area departments," European Journal of Operational Research, vol. 201, no. 1, pp. 171-182, 2010.

[11] R. Kia, A. Baboli, N. Javadian, R. Tavakkoli-Moghaddam, M. Kazemi, and J. Khorrami, "Solving a group layout design model of a dynamic cellular manufacturing system with alternative process routings, lot splitting and flexible reconfiguration by simulated annealing," Computers \& Operations Research, vol. 39, no. 11, pp. 2642-2658, 2012.

[12] R. Kia, H. Shirazi, N. Javadian, and R. T. Moghaddam, "A multiobjective model for designing a group layout of a dynamic cellular manufacturing system," International Journal of Industrial Engineering, vol. 9, no. 1, pp. 1-14, 2013.

[13] Z. Jiang, S. Zhu, H. Zhang, and Y. Wang, "Optimal acquisition and inventory control for a remanufacturing system," Discrete Dynamics in Nature and Society, vol. 2013, Article ID 120256, 7 pages, 2013.

[14] V. D. R. Guide Jr., G. C. Souza, and E. van der Laan, "Performance of static priority rules for shared facilities in a remanufacturing shop with disassembly and reassembly," European Journal of Operational Research, vol. 164, no. 2, pp. 341-353, 2005.

[15] C. Li, Y. Tang, C. Li, and L. Li, "A modeling approach to analyze variability of remanufacturing process routing," IEEE Transactions on Automation Science and Engineering, vol. 10, no. 1, pp. 86-98, 2013.

[16] Z. Jiang, H. Zhang, and J. W. Sutherland, "Development of multi-criteria decision making model for remanufacturing technology portfolio selection," Journal of Cleaner Production, vol. 19, no. 17-18, pp. 1939-1945, 2011.

[17] G. Tian, Y. Liu, H. Ke, and J. Chu, "Energy evaluation method and its optimization models for process planning with stochastic characteristics: a case study in disassembly decisionmaking," Computers and Industrial Engineering, vol. 63, no. 3, pp. 553-563, 2012.

[18] S. M. Mousavi and R. T. Moghaddam, "A hybrid simulated annealing algorithm for location and routing scheduling problems with cross-docking in the supply chain," Journal of Manufacturing Systems, vol. 32, no. 2, pp. 335-347, 2013.

[19] T. Chen and R. Xiao, "Enhancing artificial bee colony algorithm with self-adaptive searching strategy and artificial immune network operators for global optimization," The Scientific World Journal, vol. 2014, Article ID 438260, 12 pages, 2014.

[20] T. Chen and R. Xiao, "A dynamic intelligent decision approach to dependency modeling of project tasks in complex engineering system optimization," Mathematical Problems in Engineering, vol. 2013, Article ID 398123, 12 pages, 2013.

[21] R. Zhang, "A simulated annealing-based heuristic algorithm for job shop scheduling to minimize lateness," International Journal of Advanced Robotic Systems, vol. 10, article 214, 2013.

[22] N. Manavizadeh, N.-S. Hosseini, M. Rabbani, and F. Jolai, "A Simulated Annealing algorithm for a mixed model assembly U-line balancing type-I problem considering human efficiency and Just-In-Time approach," Computers \& Industrial Engineering, vol. 64, no. 2, pp. 669-685, 2013.

[23] C. Ju and T. Chen, "Simplifying multiproject scheduling problem based on design structure matrix and its solution by an improved aiNet algorithm," Discrete Dynamics in Nature and Society, vol. 2012, Article ID 713740, 22 pages, 2012. 


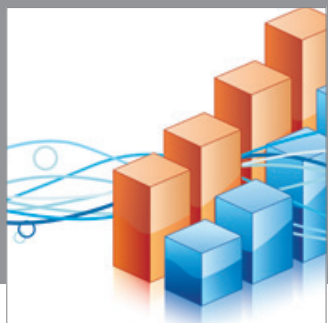

Advances in

Operations Research

mansans

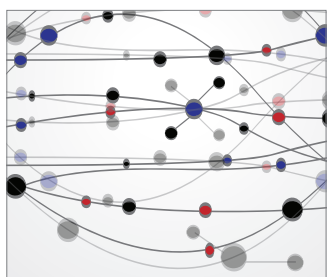

The Scientific World Journal
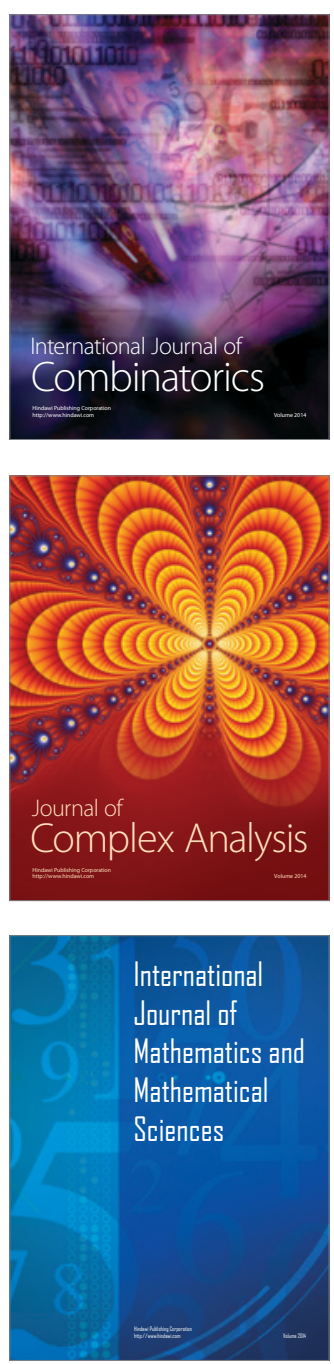
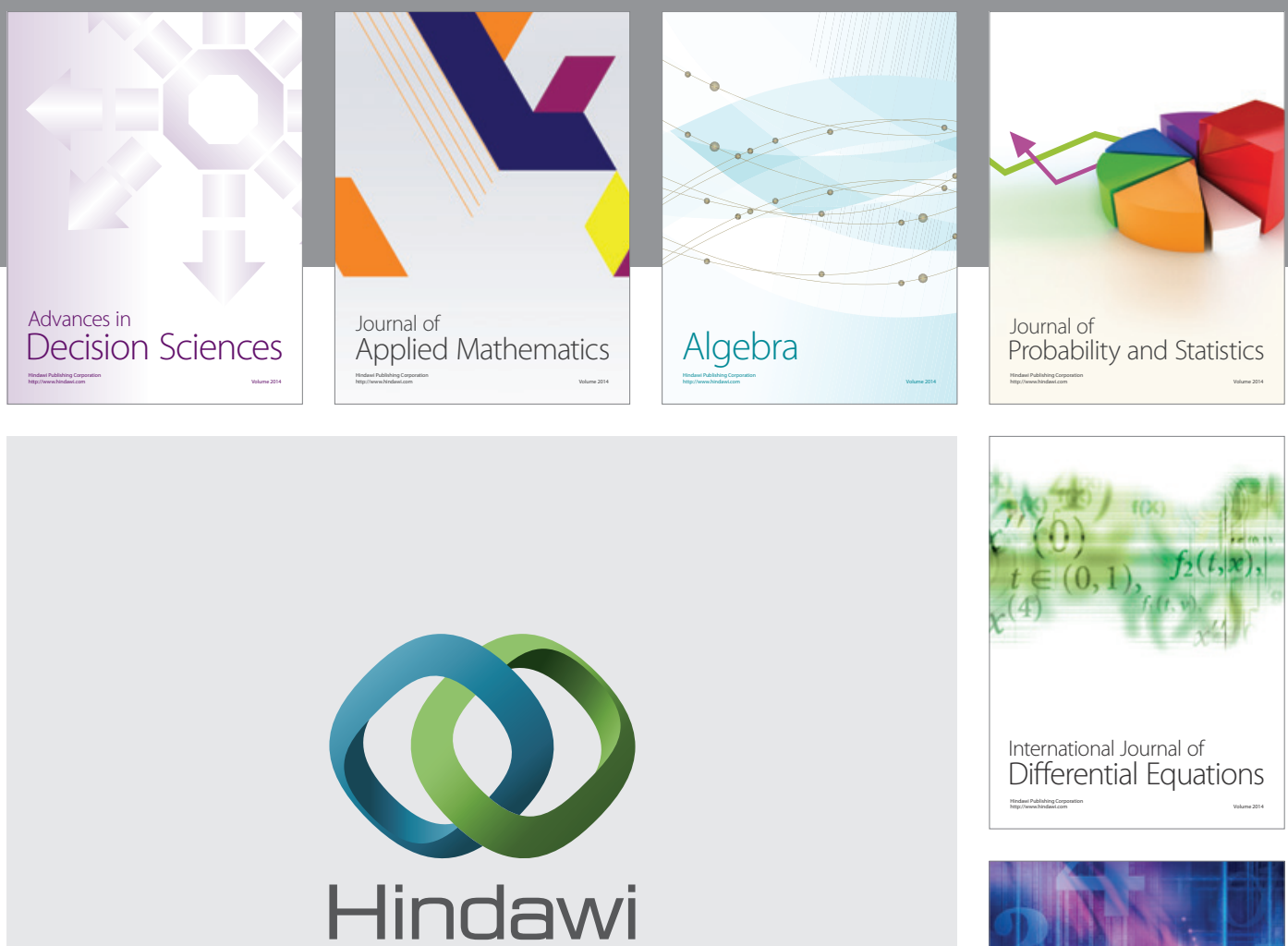

Submit your manuscripts at http://www.hindawi.com
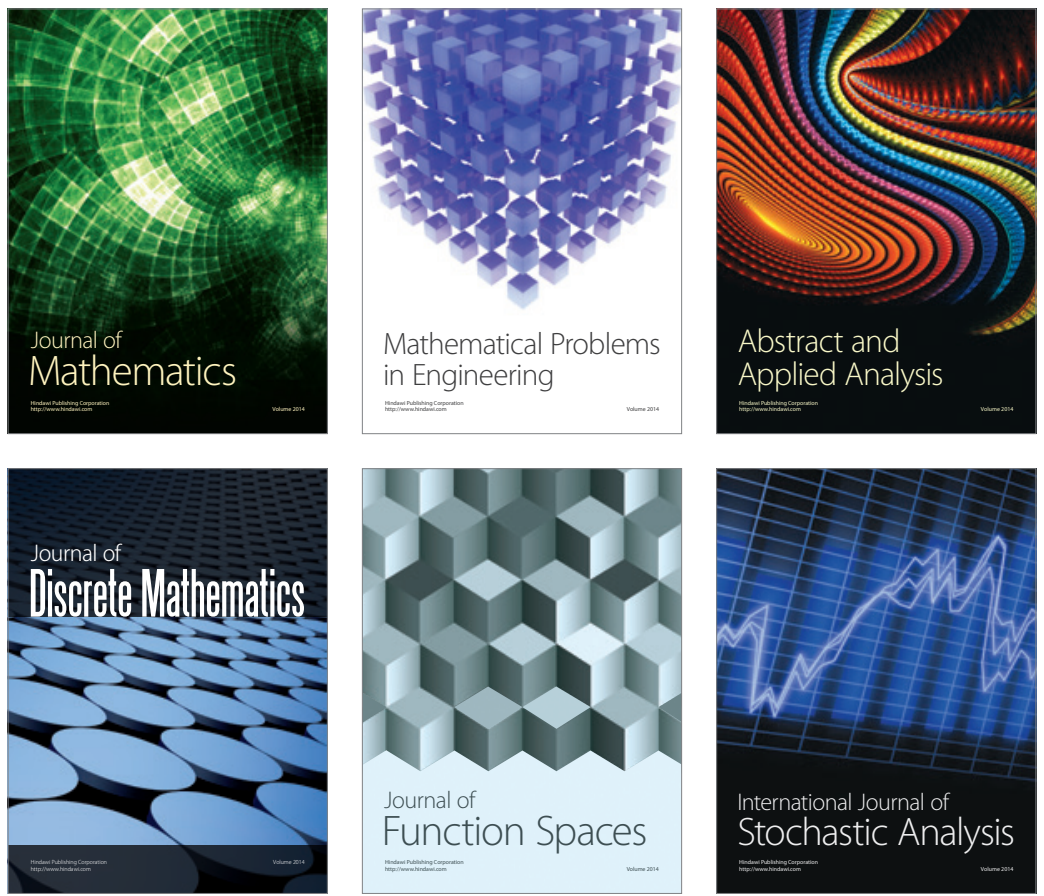

Journal of

Function Spaces

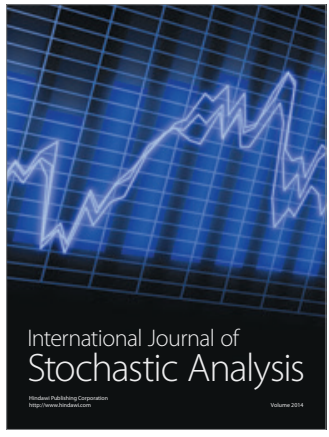

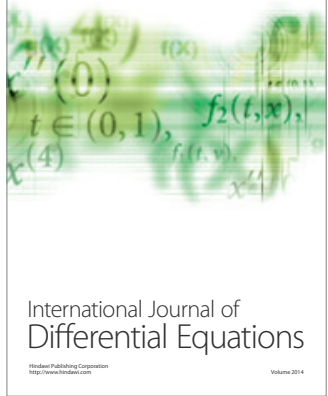
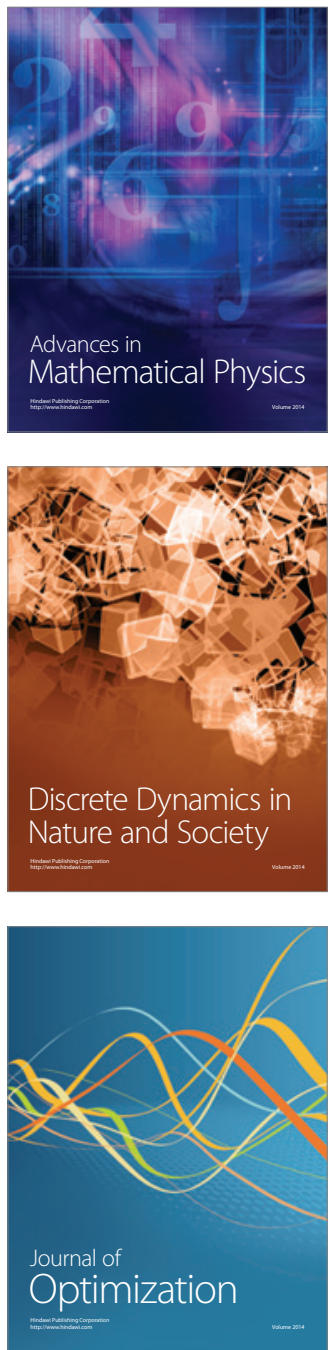